Article

\title{
Power Consumption Analysis of Electrical Installations at Healthcare Facility
}

\author{
Emmanuel Guillen-Garcia ${ }^{1}$, Angel L. Zorita-Lamadrid ${ }^{2}$, Oscar Duque-Perez ${ }^{2}$, \\ Luis Morales-Velazquez ${ }^{1, *}$, Roque Alfredo Osornio-Rios ${ }^{1}$ and Rene de Jesus Romero-Troncoso ${ }^{3}$ \\ 1 Facultad de Ingenieria, CA Mecatrónica, Universidad Autónoma de Querétaro (UAQ), Rio Moctezuma 249, \\ 76807 San Juan del Rio, Mexico; eguillen@hspdigital.org (E.G.-G.); raosornio@hspdigital.org (R.A.O.-R.) \\ 2 Department of Electrical Engineering, Universidad de Valladolid (UVa), c/Paseo del Cauce 59, \\ 47011 Valladolid, Spain; zorita@eii.uva.es (A.L.Z.-L.); oscar.duque@eii.uva.es (O.D.-P.) \\ 3 Departamento de Ingenieria Electronica, DICIS, Universidad de Guanajuato (UG), \\ Carretera Salamanca-Valle de Santiago, 36865 Salamanca, Mexico; troncoso@hspdigital.org \\ * Correspondence: lmorales@hspdigital.org; Tel.: +52-442-192-1200 (ext. 6090)
}

Academic Editor: K.T. Chau

Received: 22 November 2016; Accepted: 31 December 2016; Published: 6 January 2017

\begin{abstract}
This paper presents a methodology for power consumption estimation considering harmonic and interharmonic content and then it is compared to the power consumption estimation commonly done by commercial equipment based on the fundamental frequency, and how they can underestimate the power consumption considering power quality disturbances (PQD). For this purpose, data of electrical activity at the electrical distribution boards in a healthcare facility is acquired for a long time period with proprietary equipment. An analysis in the acquired current and voltage signals is done, in order to compare the power consumption centered in the fundamental frequency with the generalized definition of power consumption. The results obtained from the comparison in the power consumption estimation show differences between $4 \%$ and $10 \%$ of underestimated power consumption. Thus, it is demonstrated that the presence of harmonic and interharmonic content provokes a significant underestimation of power consumption using only the power consumption centered at the fundamental frequency.
\end{abstract}

Keywords: energy consumption estimation; power quality; data acquisition; data analysis

\section{Introduction}

The IEEE standard 1459-2010 [1] sets the norm to estimate power quality (PQ) indexes like power consumption, active power, reactive power, and apparent power. The standard establishes that the estimation of these indexes can be done, in first instance by using the fundamental frequency of the power line, but also mention that the estimation would be done using all frequencies, including the influence of harmonics and interharmonics. Nowadays, commercial equipment power consumption estimates are made using only the fundamental frequency, this work shows how significant the difference is between using only the fundamental frequency in contrast to using all the signal frequencies.

Additionally, hospitals and healthcare facilities have one of the highest priorities in the power supply of public buildings, as uninterrupted power supply is essential in these facilities. For this reason, there are different national and international standards that provide the recommended practice for the design and operation of electric systems in healthcare facilities [2-4]. Nevertheless, there still remain unexplored issues such as the power quality (PQ) analysis of the power supply as delivered from the grid and distribution inside the building, and how the PQ affects the electrical installation in general, due to the presence of harmonic content. According to the aforementioned issues, it is 
necessary to consider that in a typical hospital facility there are many nonlinear loads that distort the electrical waveform, introducing harmonics and interharmonics; basically, due to the great quantity of electronic equipment having switching power supplies [5]. Consequently, the estimation of the PQ indexes related to power consumption only according to the fundamental frequency is not as accurate as it should be, this is the case in most commercial equipment.

Many research papers are focused in the study of harmonic and interharmonic contents for electrical signals to locate the disturbance origin, to extract them from the signal, or to try to explain its origin. Saxena et al. [6] developed a methodology to identify harmonic sources in radial and non-radial power systems using harmonic measurement devices. This method can locate harmonic sources in the presence of harmonic injecting systems and it is independent of network topology. Ye et al. [7] present a method to detect the location of harmonic disturbance sources in low voltage networks; then, they apply Bayesian inference in order to consider the uncertainties of measurement values and the dynamic states of a grid. The method is more accurate when the harmonic content is higher and the performance is proportional to the measurement accuracy level. Yang and Bollen [8] show the verification of the classical model for the emission of interharmonics from a frequency converter; they proposed and verified a model in order to explain the origin of interharmonics from a wind turbine that can be injected into the grid. Apparently, the interharmonics are due to the difference between the generator-side and the grid-side frequencies of the full-power converter. Ferreira et al. [9] proposed a technique of independent component analysis for extracting harmonics from electrical signals; the method is tested using real power signals and non-linear loads to demonstrate adequacy for real-case applications. On the other hand, several studies are dedicated to analyzing the power consumption estimation in public or residential buildings through the use of acquired data by common commercial equipment in diverse time periods for: days, weeks, or even a year. Mahmoud et al. [10] show a case study of the electricity usage in 151 Nebraska (USA) residences, in order to prove whether the usage of real-time monitoring devices helps the participants of the study to save energy in their residences. Cherkassky et al. [11] use computational intelligence of electric power consumption in commercial buildings in order to improve the prediction accuracy of power consumption and to make contracts with utility companies according to electric load forecasting. Ortega and Manana [12] reviewed the energy use in airports, in order to analyze the main behaviors and energy trends at airports in more recent research. Babar et al. [13] developed an energy optimization technique to be applied in households in response to the dynamic behaviors of customers, this technique was intended to minimize the end user electricity bill. In other related works, a power consumption study was applied to improve residential consumer bills, proposing smart-home community architecture in power systems [14], or through the installation of energy storage systems [15]. Additionally, some investigations were conducted to analyze power consumption data in non-residential buildings, including hospitals. Christiansen et al. [16] presented a methodological approach, for a more accurate assessment of the electrical energy demand of medical equipment operated within modern hospital laboratories, to verify that the medical equipment is responsible for high electricity consumption in hospital laboratories, and found that only a few plug load groups contributed to the greater part of the total electrical energy demand. Bagnasco et al. [17] developed a load forecasting model to forecast electrical consumption a dayahead in the Cellini medical clinic of Turin, Italy. They concluded that the electrical load forecasting and modeling is an essential component for better dynamic management of the thermal behavior of buildings for energy saving policy. Boschiero [18], works with annual energy demand profiles to develop a methodology as a tool to create high-efficiency solutions, and thus predict the performance of an engine tri-generation system in a hospital. The energy consumption model presented by Zorita el al. [19], helps hospital managers to compare the energetic performance of all the installations under their responsibility in the region of Castilla and Leon (Spain). Their model defines an efficiency index to compare the energy consumption of buildings with similar functions and programs the necessary changes to optimize its energy consumption. All the aforementioned works are focused on analyzing power consumption 
information according to the standard of using only the fundamental frequency of the power line, to develop procedures or techniques for improving energy management or consumption in buildings. However, in these works, they did not analyze how the power consumption is estimated or how the presence of nonlinear loads and power quality disturbances (PQDs) in the electrical installation can affect this estimation of power consumption.

Moreover, the harmonic content of the power supply and the presence of PQDs are not only a problem for the accurate estimation of power consumption, but they are also a problem, especially in large electric installations, for calculating the specifications of protections and wiring [20], which can have a significant impact on safety. A major example of this is hospitals, because they are critical public facilities that require a detailed analysis on the PQ indexes of the power supply to prevent failures in the electrical installation. A miscalculation on the protections in a hospital electrical installation can producefailure or malfunction on lifts, escalators, lighting, or electromedical equipment, with dangerous consequences like overheating of the electrical installation that could provoke fire or damage the electronic equipment [21]. Thus, it is necessary make analyze how the actual commercial measure equipment estimates power consumption and determine if they consider the contribution of harmonics, interharmonics, and PQDs in general.

This paper presents a methodology for power consumption estimation in a hospital considering harmonics, interharmonics, and PQDs. Then, this estimation is compared to the estimation using only the fundamental frequency of the supply line. The approach of using only the fundamental frequency is the method typically implemented with commercial equipment. The data acquisition is done with proprietary equipment that has the capability of acquiring a large quantity of data for a long period of time. Extensive experimentation is conducted in a hospital facility by recording electrical signals with specialized data acquisition systems in several points of interest. Afterwards, the data are processed to estimate the power consumption, first estimating the power consumption related to the fundamental frequency, and then estimating the power consumption considering harmonics and interharmonics. Both estimations are compared and some conclusions are outlined regarding the power consumption underestimation by commercial equipment.

\section{Theoretical Background}

\subsection{Generalized Electric Power Consumption Definition}

The instantaneous power $p(t)$ on a dynamic system is defined as the rate of change at which work $W$ is done, given by,

$$
p(t)=\frac{d W}{d t}
$$

In electric circuits, the instantaneous power is obtained by the product of the instantaneous values of voltage $v(t)$ and current $i(t)$ :

$$
p(t)=v(t) \times i(t) .
$$

In general, the instantaneous power expression in alternate current circuits is a time-varying function. However, the mean value of $p(t)$ is very important in practice, because it represents the net value of the consumed power from the electromechanical conversion point of view. Therefore, the true active power as the average value of the instantaneous power over a time interval $T$ is given by,

$$
P=\frac{1}{T} \int_{0}^{T} p(t) d t .
$$

The active power $(P)$ unit in the international system is the watt $(W)$. 
The discretization of the Equation (3) results in:

$$
P_{t}=\frac{1}{T} \sum_{n=1}^{k} v_{n} \cdot i_{n}
$$

where $k$ is the number of samples, $v_{n}$ and $i_{n}$ are the $n$-th voltage and current sample respectively.

\subsection{Fundamental Definition of Power Consumption}

The IEEE 1459 standard, considers a sinusoidal voltage source as stated in (5) with a linear load having a sinusoidal current as stated in (6),

$$
\begin{gathered}
v(t)=\sqrt{2} V \sin (\omega t), \\
i(t)=\sqrt{2} I \sin (\omega t-\theta),
\end{gathered}
$$

where $V$ is the root mean square (RMS) value of the voltage, $I$ is the RMS value of the current, $\omega=2 \pi f$ is the fundamental frequency, $t$ is time, and $\theta$ is the phase angle between voltage and current waveforms at the fundamental frequency.

According to the IEEE 1459 standard, to determine the power consumption in a three-phase system, it is necessary to calculate the active power as follows:

$$
P_{1}=\frac{1}{a T_{0}} \int_{\tau}^{\tau+k T_{0}} p(t) d t .
$$

where $p(t)$ is the $v(t) \cdot i(t)$ product, $T_{0}$ is the period of one cycle (s) of the fundamental frequency, $a$ is an integer, and $\tau$ is the moment when the measurement process starts. Applying (7) to the instantaneous power definition with voltage and current as in (5) and (6) respectively, the next equation is obtained

$$
P_{1}=V I \cos (\theta) .
$$

Following the standard, $P_{1}$ must be calculated centered at the fundamental frequency. The manufacturers of the measurement equipment implement their own estimation algorithms according to the aforementioned standard for power consumption [20].

\subsection{Harmonic Definition of Power Consumption}

The harmonic and interharmonic content are elements located in frequencies out of the fundamental frequency in an electrical signal. These elements should be considered in the calculation of power consumption due to their energy input to the electrical signal. To determine the non-fundamental active power is necessary to follow (9)

$$
P_{H}=V_{0} I_{0}+\sum_{h \neq 1} V_{h} I_{h} \cos \theta_{h}=P_{t}-P_{1} .
$$

where $V_{0}$ and $I_{0}$ are the direct voltage and current respectively, $h$ is the number of the harmonic component. The calculation of $P_{H}$ includes the presence of interharmonics and subharmonics [4].

In electrical installations where the voltage and the current are sinusoidal, the estimation of the power consumption following (8) is equal to the estimation in the generalized expression from (3). However, it is expected that if the voltage source is non-sinusoidal or if the load is nonlinear, the estimation of the power consumption produces different results depending on whether the generalized or the sinusoidal expressions are used. 


\section{Methodology}

The main purpose of this work is to show the underestimation in power consumption as a result of the estimation using only the fundamental frequency when compared to the estimation using the generalized power consumption definition. In order to obtain significant results, it is necessary to estimate the power consumption over a long period of time, which requires acquiring huge amounts of data. To attend this requirement, a proprietary data acquisition system (DAS) with an integrated data logger is developed for acquiring and collecting data from the hospital electrical installations. The DAS is non-intrusive and it is easily attached to the desired three-phase power line to acquire instantaneous voltages and currents, preserving their original waveforms at the point of interest with a storage capacity of over seven days of continuous acquisition. The developed DAS is first calibrated using reference equipment. Then, the methodology is validated in a controlled installation facility by acquiring data with the developed DAS and the reference. Afterwards, two DAS units are installed in two points of interest at a hospital installation to acquire the three-phase voltage and current. Once acquired, the data is processed to estimate the power consumption around the fundamental frequency and also according to the definition of power consumption considering the signal original waveform; then both estimations are compared.

In order to verify the underestimation of the power consumption with a higher presence of harmonic content, the signal from an entire day is injected with double the original amount of harmonic and interharmonic content to simulate the increment of nonlinear loads in the electrical installation. Finally, the same methodology is applied to obtain the estimation difference.

\subsection{Calibration}

The calibration process of the DAS is depicted in the flow diagram of Figure 1. First, the proprietary DAS is placed in parallel with a reference to acquire three-phase voltages $v_{a}, v_{b}, v_{c}$ and currents $i_{a}$, $i_{b}, i_{c}$ of a test bench consisting in three balanced, star-connected loads. The first load bank is purely resistive, the second load bank is purely capacitive, and the third load bank is mostly inductive by connecting an AC motor as a load. The power consumption estimation is done according to (7) at each phase for a time stamp of one minute. The power consumption estimated by the DAS compared to the reference and from the statistical analysis of the acquired data in both systems; the calibration error (Err) of the DAS are estimated according to

$$
E r r=\sqrt{\frac{\sum d^{2}}{n}} \cdot 100 \%
$$

where $d$ is the difference between the reference value and the obtained value and $n$ the number of samples. For the calibration process, the DAS estimates the power consumption by applying the standard stated in (7).

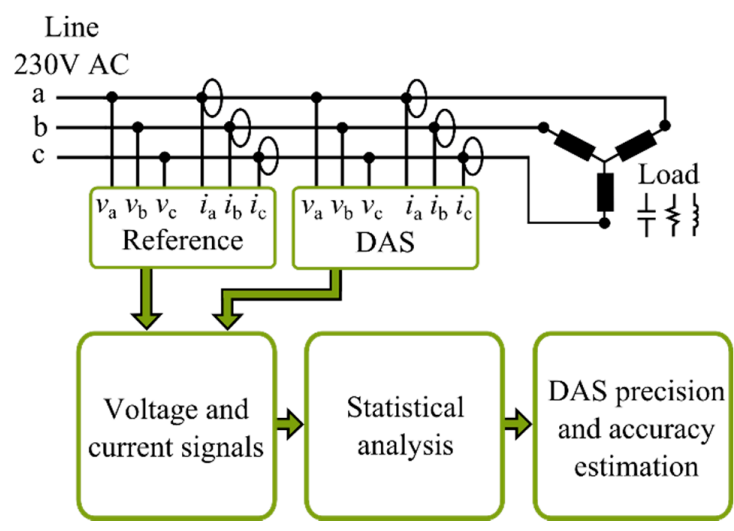

Figure 1. Calibration flow diagram. 


\subsection{Validation}

During the validation process, the calibrated DAS is placed in parallel with the reference equipment to acquire three-phase voltages and currents of real signals in a secondary electrical distribution board. Several switching power supplies, AC motors, and load plugs are connected to this distribution board. The data acquisition time window for this test is $24 \mathrm{~h}$. The acquired data in this test is separated in time stamps of one minute and the power consumption is estimated according to (4). The power consumption estimation from the DAS is compared to the estimation given by the reference to validate the functionality of the developed DAS. For the validation process, the comparison of the results between the DAS and the reference is done with the DAS using the standard specification around the fundamental frequency.

\subsection{Proposed Methodology}

The acquired data is processed with two different methods in order to compare them and show the difference between estimating power consumption as the commercial equipment around the fundamental frequency and with the whole signal waveform acquired with the proprietary equipment. The flow diagram of Figure 2 depicts the proposed methodology when the acquired data are processed in time stamps of one minute.

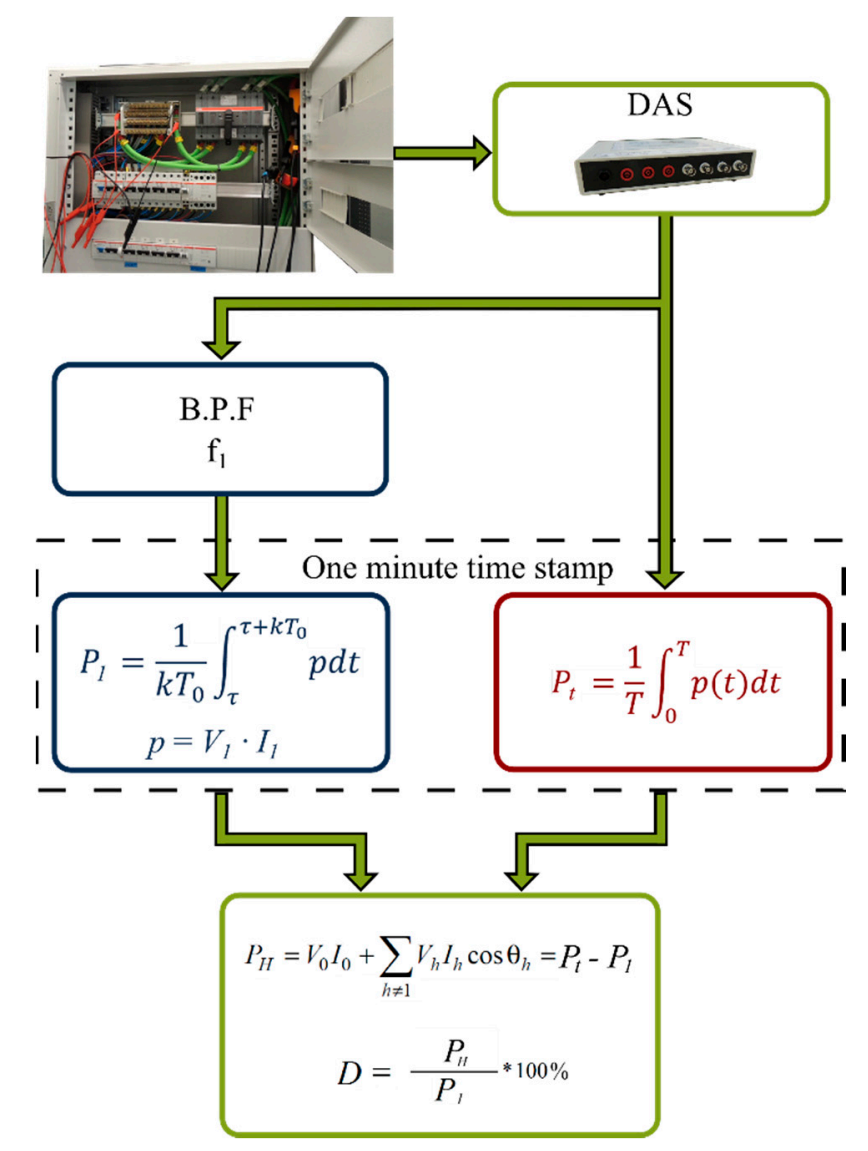

Figure 2. Flow diagram of the proposed methodology.

The first part of the method consists in processing the data according to the fundamental power consumption. This is done by applying a band-pass filter (BPF) centered on the fundamental frequency $f_{1}$ over voltage and current signals. This way, a filtered voltage signal $\left(V_{1}\right)$ Figure 2 and filtered current signal $\left(I_{1}\right)$ Figure 2 are obtained. Then, $V_{1}$ and $I_{1}$ are used in (7) to estimate the active power. The result is the estimation according to the standard centered in the fundamental frequency. 
The second part of the method consists in estimating the power consumption according to (4) using the voltage and current without filtering, and preserving their original waveforms including all possible signal disturbances.

Afterwards, the deviation percentage $D$ of the power consumption estimation $P_{t}$ is calculated using the definition in (4) relative to the power consumption estimation from the standard $P_{1}$ as follows

$$
D=\frac{P_{t}-P_{1}}{P_{1}} \cdot 100 \%,
$$

According to (9), it is possible to rewrite (11) as follows

$$
D=\frac{P_{H}}{P_{1}} \cdot 100 \%
$$

This deviation shows the underestimated power consumption of the definition against the standard where, if $P_{H}$ grows, the difference $D$ is greater.

\section{Experimental Setup}

The experimentation is done in three different stages: the first one is dedicated to the DAS calibration, the second one to validate the DAS, and the third one for the healthcare facility data test.

The DAS used in this work is able to acquire data from seven simultaneous channels at 8000 samples per second (SPS) with a 16-bit resolution. Four channels are dedicated to 1-1000 A current measurements in several available ranges and three to 100-600 V voltage measurements, also in several ranges, which depend on the settings at the point under test. The DAS includes a Texas Instruments ${ }^{\circledR}$ analog-to-digital converter ADS130E08. Unlike the reference equipment, the DAS stores all the waveforms of voltage and current signals during a long period of time using a portable memory device. This device is a standard micro SD card of $128 \mathrm{~GB}$, capable of storing up to 10 days of data. Furthermore, the SD card can be replaced when it is full, extending the storage capacity to months or even years.

The reference commercial instrument used in this work is a FLUKE 434 three-phase power analyzer [22] with FLUKE i400s AC current clamps.

The implement filter for the acquired signals is an IIR band-pass sixth-order Butterworth filter, centered at the $\mathrm{f} 1$ frequency. This choice is because the Butterworth approximation has a monotonic function in the pass-band and suppression-band. Furthermore, the recursive filters provide high attenuation in the suppression-band.

\subsection{Calibration}

The test bench with all its components for the calibration process is depicted in Figure 3. Three different load stages are used for calibration; the first stage with three-phase purely resistive loads of $71 \Omega$, the second one with three-phase purely capacitive loads of $75 \mu \mathrm{F}$, and the third one with three-phase mostly inductive loads of $560 \mathrm{mH}$. The loads are connected to a $50 \mathrm{~Hz}, 230 \mathrm{Vac}$ supply. The test time for every stage is set to $10 \mathrm{~min}$, where the DAS and the reference instruments are working simultaneously. The acquired data are processed to obtain the mean and standard deviation of the DAS regarding the reference commercial instrument to estimate the error of the DAS in order to calibrate it. 


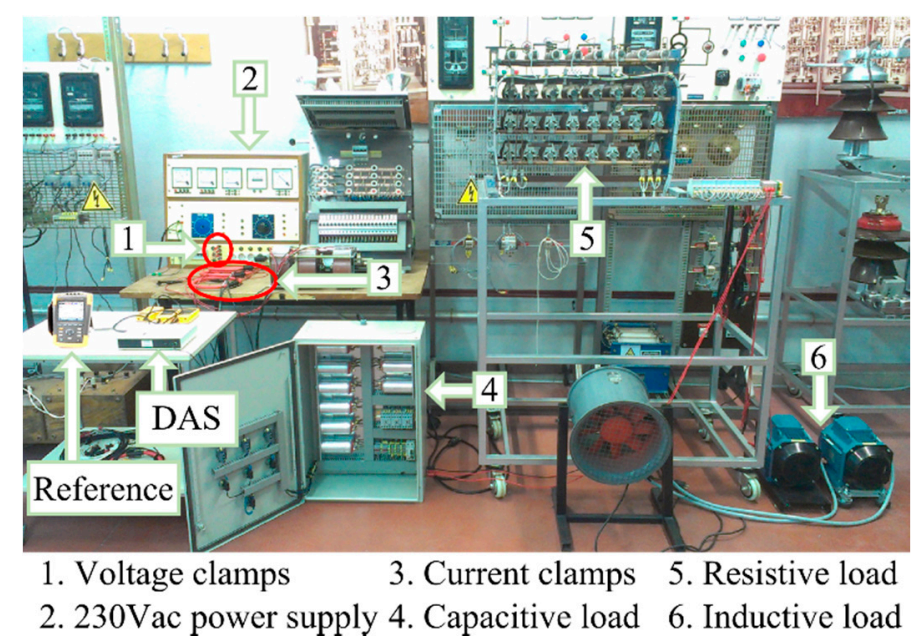

Figure 3. Test bench used for calibration.

\subsection{Validation}

For the validation process, the DAS is placed in parallel with the reference to acquire data from a secondary distribution board of an industrial electrical network as shown in Figure 4. The test consisted of the acquisition of three-phase voltage and current signals with both instruments over $24 \mathrm{~h}$ with normal activity in the electrical installation. The attached loads to the installation consist in roughly: seven switching power supplies, four AC motors of $0.75-2.2 \mathrm{~kW}$, and several plug loads, which are operated at different periods of time during the validation test.

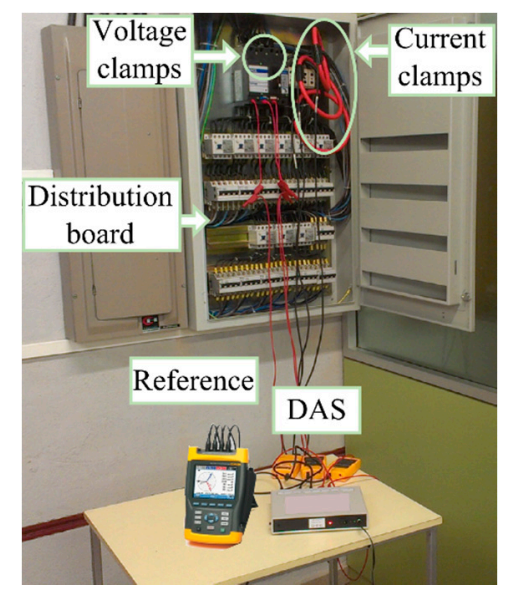

Figure 4. Distribution board for the validation test.

\subsection{Healthcare Facility Data Acquisition}

Once the DAS has been validated, the next step is to use it for acquiring real operating electrical conditions at a hospital installation in the region of Castilla y Leon (Spain) with over 600 beds.

Data acquisition at the hospital is done on a weekly basis in a few load distribution boards in order to cover the different electrical activity along the electrical installation. In this way, it is possible to capture the different load variations produced or caused by lamps, switching power supplies, plug loads, or specialized medical equipment due to their activity.

For the data acquisition, two DAS were installed in the main distribution board D3 as shown in Figure 5. One DAS is placed at the main board D3, and the other one is placed at the secondary board SB3.1.1 dedicated to supply common zones in level 1. Every DAS acquires the voltages and 
currents of the three-phase electrical installation. Figure 6 depicts the DAS installation in a typical secondary board.

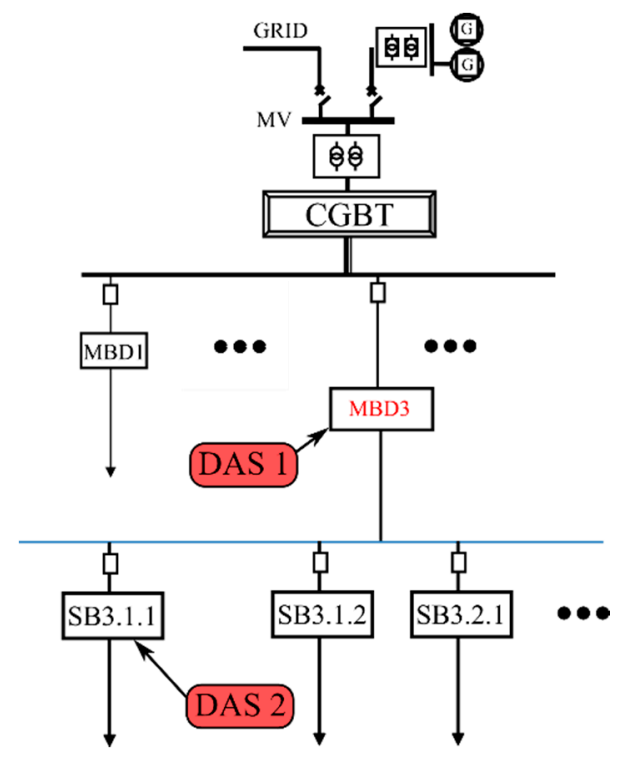

Figure 5. Data acquisition system (DAS) locations at the hospital.

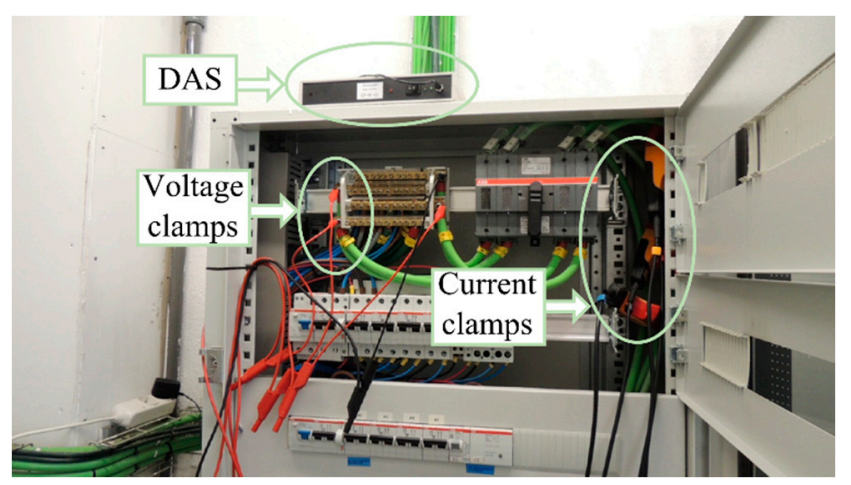

Figure 6. DAS installation at a secondary board in the hospital.

To verify that the increment in the harmonic power increases the difference in the power consumption estimations, an increment of harmonic content is simulated, removing the fundamental frequency with a zero phase filter from the original signal and then adding and normalizing the result to the original. A signal with double the harmonic and interharmonic contents is obtained.

\section{Results}

\subsection{Calibration Results}

The measurement error resulting from the calibration process in the three-phase load test bench is of $1.9 \%$ for phase $1,0.61 \%$ for phase 2 , and $0.98 \%$ for phase 3 . According with the estimated error, a mean ratio between the power consumption estimation of the reference and DAS is obtained. This mean ratio of 0.9825 is the adjustment factor for the gain in the DAS. Applying the adjustment factor in the DAS, the measurement error is reduced to $0.04 \%$ for phase $1,0.04 \%$ for phase 2 , and $0.03 \%$ for phase 3. 


\subsection{Validation Results}

Figure 7 shows the power consumption estimations obtained from the 24-h test in the three-phase industrial electrical network. Figure 7a shows the power consumption estimation for the reference and DAS in phase 1 , with $0.008 \%$ of average deviation percentage between the proprietary DAS and the Fluke 434 reference; Figure $7 \mathrm{~b}$ shows phase 2 with $0.013 \%$ of average deviation percentage, and Figure $7 \mathrm{c}$ shows phase 3 with $0.01 \%$ of average deviation percentage. The sudden power consumption increases in the all phases are due to the on-off action at different times of the elements connected to the electrical network, mainly AC motors. The increment in the power consumption of phase 3 is due to the continuous operation of switching power supplies.

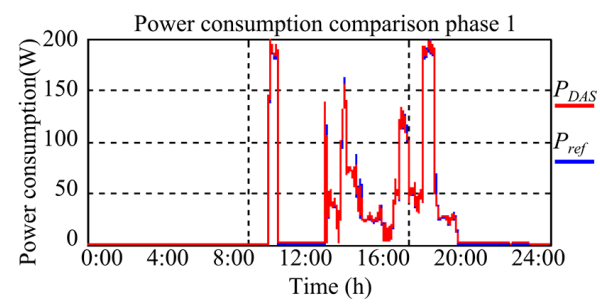

(a)

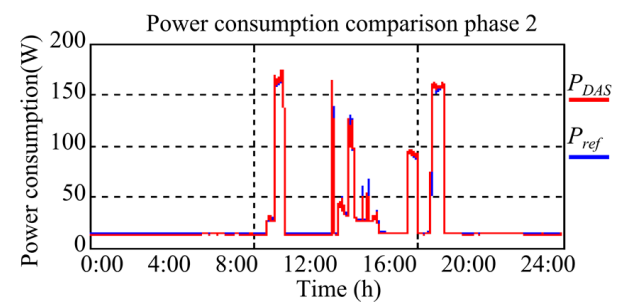

(b)

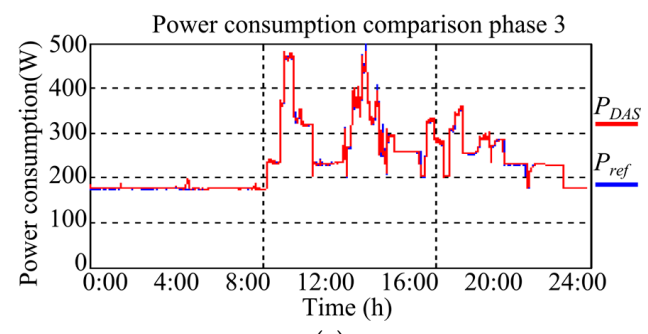

(c)

Figure 7. Validation results (a) phase 1; (b) phase 2; and (c) phase 3.

\subsection{Healthcare Facility Data Acquisition Results}

Figure 8 shows the results obtained from the hospital during $24 \mathrm{~h}$ of data acquisition, where the Figure 8a shows the comparison between the power consumption estimation around the fundamental frequency, and the estimation according to the definition with the original waveform during one day in the main board D3 (MBD3), in this case the average of the deviation percentage between the estimation from the definition to the estimation from the fundamental frequency is $4.29 \%$, which is equivalent to $0.58 \mathrm{~kW}$ of underestimated power consumption per minute, with a standard deviation of $0.02 \%$, equivalent to $2.7 \mathrm{~W}$. Figure $8 \mathrm{~b}$ shows the same comparison between power consumption estimations for the secondary board 3.1.1 (SB3.1.1), in this case, the average of the deviation percentage is $4.36 \%$ equivalent to $0.28 \mathrm{~kW}$ of underestimated power consumption per minute, with a standard deviation of $0.44 \%$, equivalent to $28.25 \mathrm{~W}$.

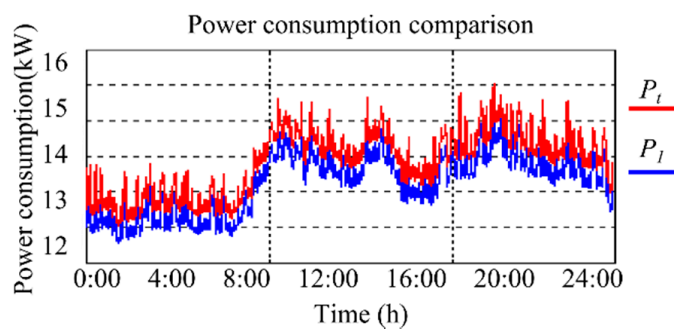

(a)

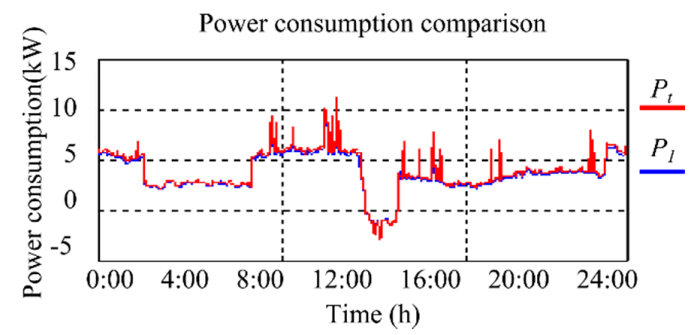

(b)

Figure 8. Power consumption difference estimation in: (a) main board MBD3; and (b) secondary board SB3.1.1. 
The aforementioned difference in the power consumption estimations is due to the presence of significant PQD. In the case of the main board MBD3, Figure 9 depicts the voltage and current signals at a given period of time (13:00 h). Figure 9 a shows the original waveforms for the voltage and current where the presence of stationary disturbances distorting the current waveform can be seen. On the other hand, Figure $9 \mathrm{~b}$ shows the filtered voltage and current signals used for the estimation of the power consumption according to the fundamental frequency. The presence of PQD makes the estimation of the power consumption according to the definition to be greater than the estimation according to the fundamental frequency $4.29 \%$, which must be taken into account to determine the specifications of the electrical components for this transmission line. Figure 10a shows the FFT spectrum for the original current signal, where the fundamental frequency is at $50 \mathrm{~Hz}$, and all the frequency components correspond to odd harmonics generated due to the PQD presence in the signal; these harmonics represent the $22.46 \%$ of the current signal. On the other hand, Figure 10b shows the FFT spectrum for the current signal as the commercial equipment does; in this case, the harmonic components do not exist because the PQDs are filtered out from the signal.

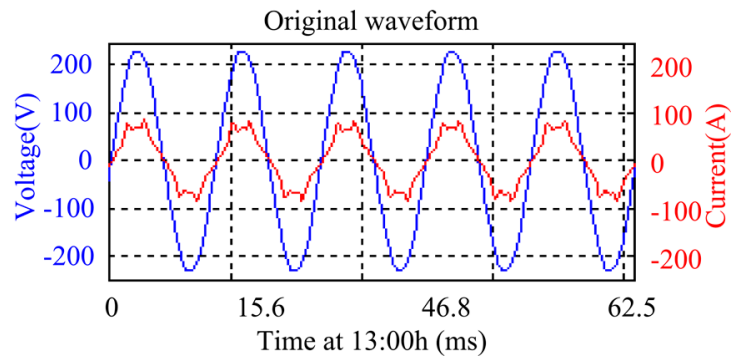

(a)

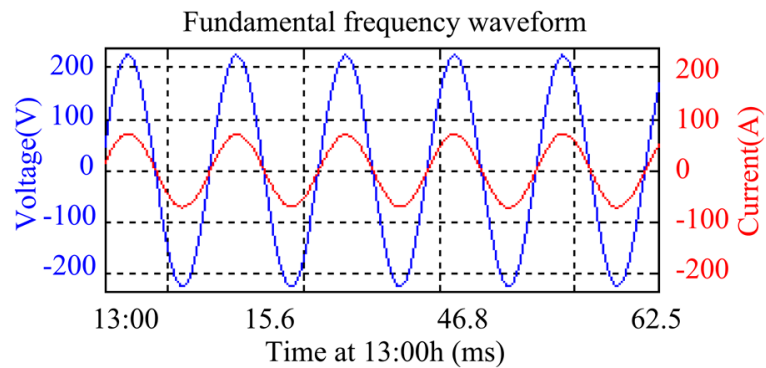

(b)

Figure 9. Main board MBD3 voltage and current signals at a time when stationary disturbances are present: (a) original signals; and (b) filtered signals around the fundamental frequency.

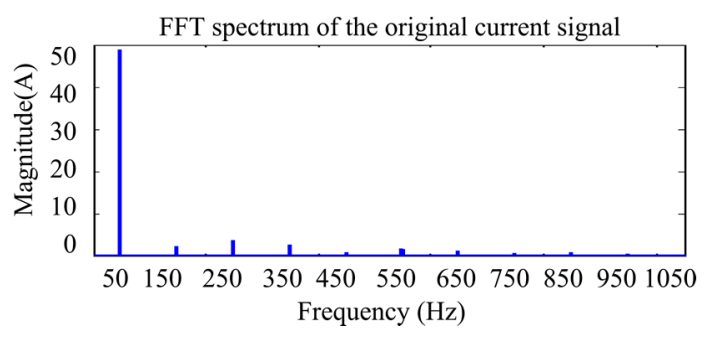

(a)

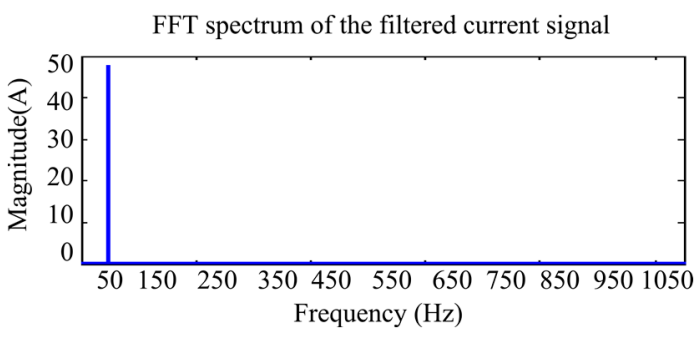

(b)

Figure 10. Main board MBD3 FFT spectrum at a time when stationary disturbances are present: (a) original current signal spectrum; and (b) filtered current signal around the fundamental frequency.

For the secondary board SB3.1.1, Figure 11a shows the presence of severe stationary PQD in the original current signal waveform during the hour 14:00. Figure 11b depicts the voltage and current signals after filtering around the fundamental frequency. Figure 12a shows the FFT spectrum of the original current signal; in this case, the odd harmonic content is more evident because the stationary PQDs are more severe than the ones in the MBD3. These harmonics represent $55.36 \%$ of the current signal. Figure $12 \mathrm{~b}$ shows the FFT spectrum for the current signal according to the standard, where the harmonic content is mainly filtered out. 


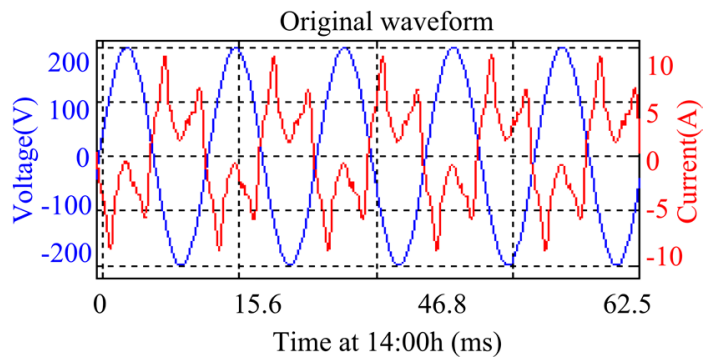

(a)

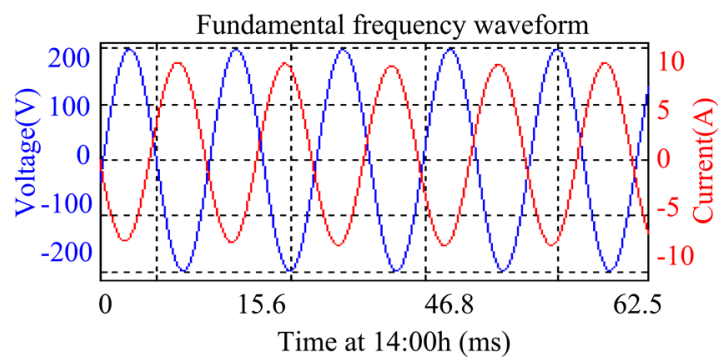

(b)

Figure 11. Secondary board SB3.1.1 voltage and current signals at a time when severe stationary disturbances are present: (a) original signals; and (b) filtered signals around the fundamental frequency.

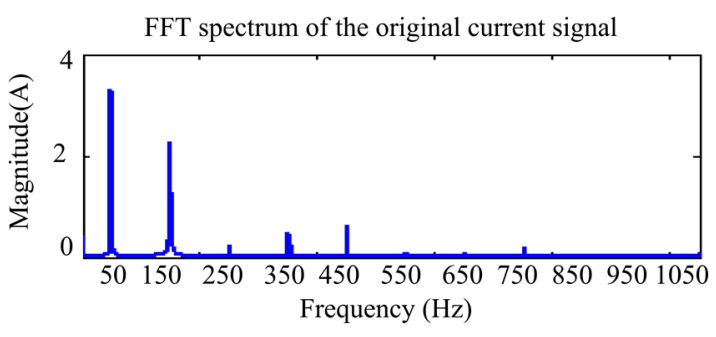

(a)

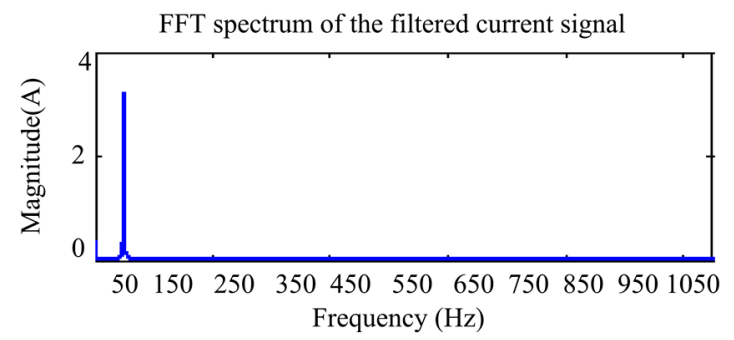

(b)

Figure 12. Secondary board SB3.1.1 FFT spectrum at a time when severe stationary disturbances are present: (a) original current signal; and (b) filtered current signal around the fundamental frequency.

The standard deviation on the estimation of the power consumption for a whole day at the secondary board SB3.1.1 is 0.44 , which is higher than the standard deviation at the main board MBD3 of 0.02. This is due to the increased presence of transient PQD at the secondary board SB3.1.1. For instance, Figure 13a depicts the original voltage and current signals at the hour 8:00 in the secondary board SB3.1.1 when one of these transient PQDs occurs. Figure 13b shows the voltage and current signals after filtering around the fundamental frequency. These transient PQDs are responsible for the power consumption underestimation when using the recommended standard estimation around the fundamental frequency.

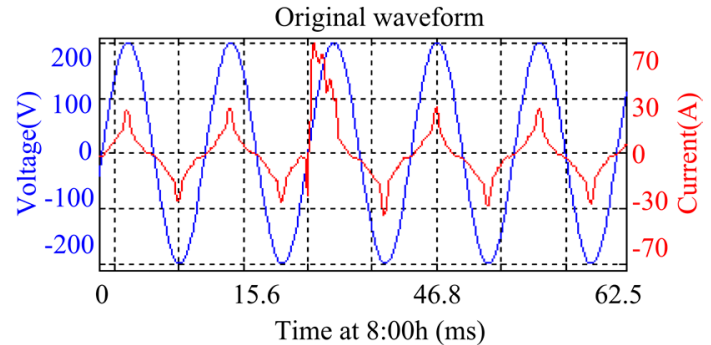

(a)

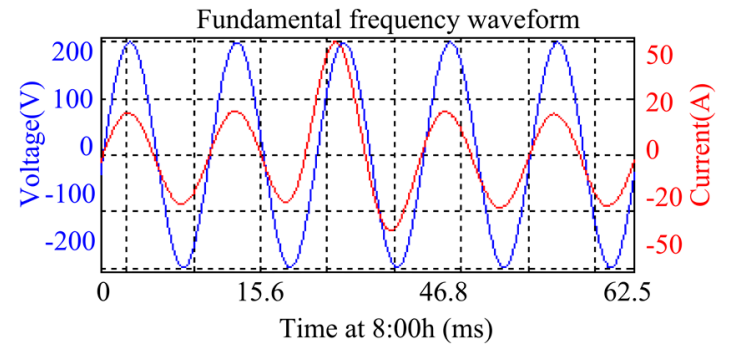

(b)

Figure 13. Secondary board SB3.1.1 voltage and current signals at a time when transient PQDs are present: (a) original signals; and (b) filtered signals around the fundamental frequency.

The simulated signal for the test with the double harmonic and interharmonic contents corresponds to the original signal shown in Figure 8b. Figure 14a shows the original signal where the underestimated power consumption deviation is of $4.29 \%$. Figure $14 \mathrm{~b}$ shows the simulated signal with the double harmonic and interharmonic contents where the underestimated power consumption deviation is $8.5 \%$. 


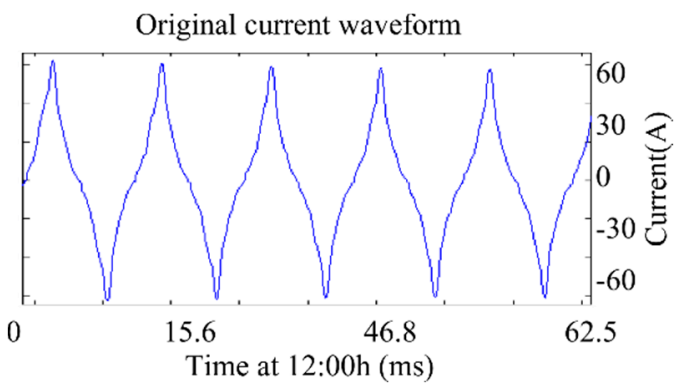

(a)

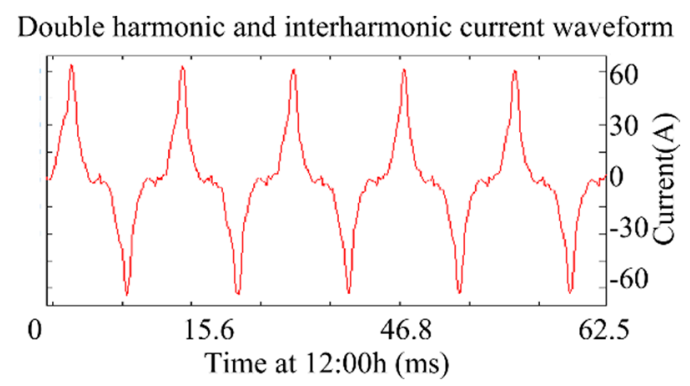

(b)

Figure 14. Current signals: (a) the original current waveform; and (b) the simulated double harmonic and interharmonic current waveform.

\section{Conclusions}

The comparative analysis for this work shows that the method used in the commercial equipment for estimating power consumption underestimates the consumption when it is compared to the estimation considering harmonics, interharmonics, and PQDs. It is possible to infer that the commercial equipment only uses the fundamental frequency in the calculation of power consumption. When the influence of PQD on the voltage and current signals are discarded, the underestimation of the power consumption using the commercial equipment method increases in presence of large disturbances. Consequently, most of the power consumption estimations made using commercial equipment could be improved in order to consider the presence of harmonic and PQD content.

Estimating power consumption with the generalized electric power definition can be more accurate than the estimation using the recommended standard around the fundamental frequency, especially in facilities where stationary and transient phenomena are expected, forcing a detailed analysis of the voltage and current signals. This is due to the power consumption estimation with the standardized form tending to underestimate between $4 \%$ and $10 \%$ the power consumption. This underestimation is more relevant in facilities where the protection estimation in the electrical installation is critical, in order to guarantee the constant energy supply and safety along the electrical installation, especially to prevent fire due to the wiring overheating.

Furthermore, the increase in the use of switching power supplies causes non-linear load increase and the PQD will be more severe. Due to this, the underestimation of power consumption by commercial equipment will increase in the future as demonstrated with the simulated signal having double harmonic contents, it is time to propose adjustments in the procedures for measuring power consumption with commercial equipment to achieve accurate measurements that consider not only the fundamental frequency. This work presents a case study focused on a health care facility, but the results can be extended to almost any electrical installation.

Acknowledgments: We would like to thank Consejo Nacional de Ciencia Y Tecnología (CONACYT) for providing economic support in this work, and to Sanidad de Castilla Y Leon (SACYL) for providing the access to health care facilities. Finally, thanks to the next three projects: SEP-CONACYT 222453-2013, FOMIX QRO-2014-C03-250269, and FOFIUAQ-FIN201613.

Author Contributions: Angel L. Zorita-Lamadrid and Oscar Duque-Perez conceived and designed the experiments; Emmanuel Guillen-Garcia performed the experiments and processed data; Luis Morales-Velazquez, Roque Alfredo Osornio-Rios, and Rene de Jesus Romero-Troncoso analyzed the data; Emmanuel Guillen-Garcia and Luis Morales-Velazquez wrote the paper.

Conflicts of Interest: The authors declare no conflict of interest. 


\section{References}

1. IEEE Standard Definitions for the Measurement of Electric Power Quantities under Sinusoidal, Nonsinusoidal, Balanced, or Unbalanced Conditions; IEEE Standard 1459-2010; The Institute of Electrial and Electronics Engineers: New York, NY, USA, 2010.

2. IEC Electrical Installations of Buildings-Requirements for Special Installations or Locations-Medical Locations; IEC International Standard; International Electric Commission: Geneva, Switzerland, 2002.

3. IEEE Recommended Practices for Electric Systems in Health Care Facilities; IEEE Standard 602; The Institute of Electrial and Electronics Engineers: New York, NY, USA, 1996.

4. Ministry of Industry, Tourism and Trade. Installations in Areas of Public Assembly, Guide BT-28; Ministerio de la Industria Turismo y Comercio: Castilla y Leon, Spain, 2002.

5. Escrivá, G.; Roldán, C.; de Jong, E. Nuisance tripping of residual current circuit breakers in circuits supplying electronic loads. Electr. Power Syst. Res. 2016, 131, 139-146. [CrossRef]

6. Saxena, D.; Bhaumik, S.; Singh, S. Identification of multiple harmonic sources in power system using optimally placed voltage measurement devices. IEEE Trans. Ind. Electron. 2014, 61, 2483-2492. [CrossRef]

7. Ye, G.; Xiang, Y.; Cuk, V.; Cobben, J. Harmonic disturbance location by applying Bayesian inference. Electr. Power Syst. Res. 2016, 140, 886-894. [CrossRef]

8. Yang, K.; Bollen, M. Interharmonic currents from a Type-IV wind energy conversion system. Electr. Power Syst. Res. 2017, 143, 357-364. [CrossRef]

9. Ferreira, D.; Nagata, E.; Ferreira, S.; de Seixas, J.; Duque, C.; Marques, C.; Guedes, J.; Cerqueira, A. Method based on independent component analysis for harmonicextraction from power system signals. Electr. Power Syst. Res. 2015, 119, 19-24. [CrossRef]

10. Alahmad, M.; Wheeler, P.; Schwer, A.; Eiden, J.; Brumbaugh, A. A comparative study of three feedback devices for residential real-time energy monitoring. IEEE Trans. Ind. Electron. 2012, 59, 2002-2013. [CrossRef]

11. Cherkassky, V.; Chowdhury, S.; Landenberg, V.; Tewari, S.; Bursch, P. Prediction of electric power consumption for commercial buildings. In Proceedings of the International Joint Conference on Neural Networks, San Jose, CA, USA, 31 July-5 August 2011.

12. Ortega, S.; Manana, M. Energy research in airports: A review. Energies 2016, 82, 92-104. [CrossRef]

13. Babar, M.; Javaid, N.; Ahmad, A.; Jamil, M.; Ali, Z.; Qasim, U.; Alrajeh, N. Energy optimization in smart homes using customer preference and dynamic pricing. Energies 2016, 74, 286-292.

14. Anees, A.; Chen, Y.-P. True real time pricing and combined power scheduling of electric appliances in residential energy management system. Appl. Energy 2016, 165, 592-600. [CrossRef]

15. Sugihuara, H.; Yokoyama, K.; Saeki, O.; Tsuji, K.; Funaki, T. Economic and efficient voltage management using customer-owned energy storage systems in a distribution network with high penetration of photovoltaic systems. IEEE Trans. Power. Syst. 2013, 28, 102-111. [CrossRef]

16. Christiansen, N.; Kaltschmit, M.; Dzukowski, F.; Isensee, F. Electricity consumption of medical plug loads in hospital laboratories: Identification, evaluation, prediction and verification. Energy Build. 2015, 107, $392-406$. [CrossRef]

17. Bagnasco, A.; Fresi, F.; Saviozzi, M.; Silvestro, F.; Vinci, A. Electrical consumption forecasting in hospital facilities: An application case. Energy Build. 2015, 103, 261-270. [CrossRef]

18. Boschiero, D. An energy and exergy analysis of a high-efficiency enginetrigeneration system for a hospital: A case study methodologybased on annual energy demand profiles. Energy Build. 2014, 76, 185-198.

19. Zorita, A.; Fernández, M.; García, L.; Duque, O. A statistical modeling approach to detect anomalies in energetic efficiency of buildings. Energy Build. 2015, 110, 377-386. [CrossRef]

20. Malhotra, R. Management of risk and economics of LV power system protection for industrial facilities. IEEE Trans. Ind. Appl. 2016, 52, 1308-1313.

21. Nazmul, A.; Taib, S.; Shawal, M.; Ishak, D. A semi-automatic approach for thermographic inspection of electrical installations within buildings. Energy Build. 2012, 55, 585-591. [CrossRef]

22. Fluke Corporation. Three-Phase Power Quality and Energy Analyzers; Fluke Co.: Everett, WA, USA, 2012.

(C) 2017 by the authors; licensee MDPI, Basel, Switzerland. This article is an open access article distributed under the terms and conditions of the Creative Commons Attribution (CC-BY) license (http://creativecommons.org/licenses/by/4.0/). 